TRABAJOS ORIGINALES

\title{
Mutaciones en la región determinante de resistencia a quinolonas (QRDR) del gen gyrA de Neisseria gonorrhoeae presente en muestras clínicas de hombres que tienen sexo con hombres
}

\section{Mutations in the Determining Region of Quinolone Resistance (QRDR) of the gyrA gene of Neisseria gonorrhoeae present in clinical samples of men who have sex with men}

\author{
Liz Sánchez Palencia ${ }^{1 *}$ y José Acosta Cáceres ${ }^{2}$
}

\author{
1 Facultad de Ciencia Biológicas, Universidad Nacional Mayor de San Marcos. Apartado Postal 11-0058, Lima 11, Perú. \\ 2 Facultad de Medicina, Universidad San Martin de Porres. Av. Alameda del Corregidor 1531, Lima 12, Perú. \\ ${ }^{*}$ Autora para correspondencia \\ Email Liz Sánchez: sanchez.palencializ@gmail.com \\ Email José Acosta: jomiacca@hotmail.com
}

\begin{abstract}
Resumen
Neisseria gonorrhoeae resistente a quinolonas (QRNG) es un problema muy importante en salud pública por su rápido desarrollo de resistencia a quinolonas, por lo que la OMS recomienda reforzar la vigilancia de resistencia antimicrobiana para orientar el tratamiento. En Perú hay pocos reportes sobre vigilancia de QRNG, y menos aún trabajos relacionados a patrones de mutación. Este estudio busca encontrar mutaciones en la Región Determinante de Resistencia a Quinolonas (QRDR) del gen gyrA de N. gonorrhoeae a partir de 1096 muestras clínicas de orina e hisopados, colectadas entre 2012 y 2013, provenientes de 367 hombres que tuvieron sexo con hombres (HSH). Se detectaron 58 muestras positivas a $N$. gonorrhoeae en $45 \mathrm{HSH}$ mediante el ensayo de APTIMA Combo 2, y 11 muestras positivas a QRNG de 11 HSH mediante PCR en Tiempo Real. Las bacterias resistentes a quinolonas fueron analizadas por secuenciamiento e identificamos que el patrón frecuente de mutación fue la doble mutación de Ser-91 a Phe y de Asp-95 a Gly en la QRDR del gen gyrA. En conclusión, estas dobles mutaciones en la secuencia QRDR del gen gyrA indicarían la presencia de N. gonorrhoeae con fenotipo resistente a quinolonas en muestras clínicas de HSH de Lima-Perú, resaltando que éste es el primer estudio hecho en Perú sobre esta población de alto riesgo.
\end{abstract}

Palabras claves: Neisseria gonorrhoeae; HSH; gen gyrA; resistencia a quinolonas.

\section{Abstract}

Neisseria gonorrhoeae resistant to quinolones (QRNG) becomes a very important problem in public health due to the development of rapid resistance to quinolones, which is why the WHO recommends reinforcing antimicrobial resistance monitoring to guide treatment. In Peru there are few reports on QRNG surveillance and still less work related to mutation patterns. This study aims to find mutations in the Determinant Region of Quinolone Resistance (QRDR) of the gyrA gene of $N$. gonorrhoeae from 1096 clinical samples of urine and swabs from 367 men who have sex with men (MSM) collected during the period 2012-2013. We detected 58 N. gonorrhoeae positive samples in 45 MSM by means of the APTIMA Combo 2 assay, and 11 QRNG positive samples of $11 \mathrm{MSM}$ by Real Time PCR. The quinolone-resistant bacteria were analyzed by sequencing and we identified that the frequent mutation pattern was the double mutation of Ser-91 to Phe and Asp-95 to Gly in the QRDR of the gyrA gene. In conclusion, these double mutations in the QRDR sequence of the gyrA gene would indicate the presence of $N$. gonorrhoeae with quinolone resistant phenotype in clinical samples of MSM from Lima-Peru, noting that this is the first study done in Peru on this high population risk.

Key words: Neisseria gonorrhoeae; MSM; gene gyrA; resistance to quinolones.

Citación:

Sánchez Palencia L. \& J. Acosta Cáceres. 2017. Mutaciones en la Región Determinante de Resistencia a Quinolonas (QRDR) del gen gyrA de Neisseria gonorrhoeae presente en muestras clínicas de hombres que tienen sexo con hombres. Revista peruana de biología 24(3): 283 - 292 (Octubre 2017). doi: http://dx.doi.org/10.15381/rpb.v24i3.13905

Información sobre los autores:

LSP realizó los procedimientos de laboratorio y JAC realizó el análisis del secuenciamiento. LSP redactó el manuscrito y JAC contribuyó a éste y lo revisó

Los autores no incurren en conflictos de intereses. 


\section{Introducción}

La gonorrea es la segunda infección de transmisión sexual bacteriana más reportada y un problema en salud pública de gran importancia (CDC 2006); ya que es una enfermedad altamente contagiosa que no sólo compromete al epitelio de la uretra, cérvix y recto, sino también a otros sitios de infección como faringe y cavidad bucal, regiones en las que se presenta como resultado del contacto orogenital (Arteaga \& Arteaga 2008). La infección inicial ocurre en las superficies mucosas del tracto genitourinario, rectal o bucofaríngeo; y el sitio de infección más frecuente es la uretra, seguido por el recto y orofaringe (Pardi et al. 2005). Hasta hace poco, ser diagnosticado con gonorrea no representaba mayor problema pues podía curarse con antibióticos. Actualmente, se considera que la gonorrea pronto estará en la lista de infecciones que no tendrán tratamiento debido a la falta de nuevas vacunas y fármacos para combatir la aparición de cepas multirresistentes en todo el mundo. Por tal motivo, desde hace más de dos décadas la OMS estableció el Programa de vigilancia de gonococos resistentes a antimicrobianos (GASP) para monitorear el surgimiento y propagación de gonococos resistentes a los tratamientos (OMS 2015).

La resistencia antimicrobiana de varios agentes patógenos sexualmente transmitidos está aumentando en muchas regiones del mundo además, las fluoroquinolonas, que al principio fueron consideradas como un régimen terapéutico recomendado, ahora son contraindicadas en algunas zonas debido a la rápida diseminación de aislados resistentes (Katz et al. 2012). Las recomendaciones de utilizar fármacos más efectivos suelen ser motivo de preocupación en cuanto al costo y al posible uso inadecuado, pues se observó que Neisseria gonorrhoeae presentó a lo largo del tiempo un incremento en la resistencia a los antibióticos usados para su tratamiento. De esta manera, $N$. gonorrhoeae resistente a quinolonas se ha propagado haciendo no aconsejable el tratamiento con quinolonas tales como ciprofloxacino en muchas áreas del mundo (CDC 2011, Unemo 2011). En los últimos años la resistencia antimicrobiana se ha incrementado y esto aunado a la falta de agentes antimicrobianos innovadores para hacer frente a estas nuevas cepas resistentes ha conllevado a la advertencia de que en pocos ańos tal vez no seamos capaces de tratar estas infecciones bacterianas. Neisseria gonorrhoeae tiene la capacidad de producir infecciones asintomáticas, lo cual crea reservorios que ayudan a diseminar su transmisión y es fundamental para su supervivencia por largo tiempo en el ser humano. La vigilancia sigue siendo la herramienta más útil para combatir la resistencia a antimicrobianos en gonorrea y esta resistencia se debe a la rápida diseminación de gonococos resistentes en varios países desarrollados y en vías de desarrollo (Stefanelli 2011).

Se han realizado estudios a nivel molecular de la enzima ADN girasa, la cual presenta dos subunidades de $\operatorname{gyr} A$ y dos subunidades de $\operatorname{gyr} B$ codificadas por los genes $\operatorname{gyr} A$ y $\operatorname{gyr} B$ respectivamente, y se determinó que un mecanismo de resistencia de Neisseria gonorrhoeae involucra mutaciones en la Región Determinante de Resistencia a Quinolonas (QRDR) del gen gyrA de la ADN girasa, lo que produce proteínas alteradas que ya no podrán ser ligadas a las quinolonas. Las quinolonas tienen un efecto bactericida cuando están ligadas a enzimas diana como la ADN girasa, la cual es esencial para la replicación del $\mathrm{ADN}$ bacteriano dentro de la célula que infecta y si la bacteria presenta dichas mutaciones en el gen $\operatorname{gyr} A$, las quinolonas son incapaces de inhibir la replicación del ADN y la bacteria llega a ser menos susceptible. Lindbäck et al. (2005) detectaron un segmento de 8 aminoácidos en la región del gen gyrA de $N$. gonorrhoeae que presentaba mutaciones en el codón de Ser 91 y Asp 95, indicando que estas mutaciones presentes en el gen gyrA están relacionadas directamente con valores de resistencia a quinolonas en ensayos de Concentración Mínima Inhibitoria (MIC). Además, se mostró también que mutaciones en la Ser91 del gen gyrA podrían servir como marcadores de resistencia a quinolonas (Zhang et al. 2009).

La gonorrea es una infección de presentación mundial y la bacteria $N$. gonorrhoeae resistente a las quinolonas (QRNG) es frecuente en muchas regiones de Asia y el Pacífico. La gonorrea es considerada una de las infecciones de transmisión sexual más frecuentes en diversas partes del mundo; la OMS considera que anualmente unos 500 millones de personas contraen alguna de las 4 ITS más reconocidas entre éstas 106,1 millones de casos para la gonorrea (Soto-Cáceres 2015).

En el Perú no se cuenta aún con un Sistema de Vigilancia Nacional como sí lo hay para otras ITS como el VIH y la sífilis. Para gonorrea sólo hay pequeños grupos de monitoreo mensual de casos positivos para gonococo en secreciones cervicales de trabajadoras sexuales. En el año 2002, las diferentes direcciones de salud del país reportaron 1229 cultivos positivos de gonococo en 92752 flujos vaginales, dando un resultado esperado nacional de $1.3 \%$ de cultivos positivos en secreciones cervicales de trabajadoras sexuales; sin embargo en nuestro país no existe ningún trabajo sobre detección de QRNG en hombres que tienen sexo con hombres (HSH), el cual es un grupo de riesgo que presenta una frecuencia elevada de infección por $N$. gonorrhoeae.

Las quinolonas son consideradas como drogas de primera línea por el Centro de Control y Prevención de Enfermedades (CDC 2011) y por el Ministerio de Salud de nuestro país (CONAMUSA-MINSA 2006) para el tratamiento de infecciones producidas por $N$. gonorrhoeae. Sin embargo, a pesar de la rápida propagación de resistencia antimicrobiana de esta bacteria a nivel mundial, en nuestro país hay poca información sobre $N$. gonorrhoeae resistente a fluoroquinolonas debido a la falta de trabajos de investigación enfocados a identificar QRNG y al difícil cultivo de esta bacteria, pues los gonococos son de crecimiento lento y con requerimientos nutricionales estrictos; ambos aspectos son obstáculos en la investigación y tratamiento eficaz contra esta bacteria.

Por lo mencionado, el presente estudio está orientado a la detección de QRNG e identificación de mutaciones en la Región Determinante de Resistencia a Quinolonas (QRDR) del gen gyrA de QRNG mediante métodos moleculares. De esta forma consideramos los resultados del presente estudio como una gran contribución para incentivar la vigilancia y control de $N$. gonorrhoeae y permitir una terapia antibiótica oportuna a los pacientes afectados impidiendo la transmisión e incremento progresivo de estas bacterias resistentes a quinolonas.

\section{Material y métodos}

Colección de muestras.- Entre los años 2012 y 2013, un total de 1096 muestras de orina y de hisopados rectales, faríngeos, uretrales fueron colectados de 367 hombres que tienen sexo con hombres (HSH), cuyo rango de edad era de 18 a 45 ańos y que informaron haber tenido relaciones sexuales orales o anales 
con otro varón en los 12 meses anteriores al estudio. De las 1096 muestras se obtuvo 383 muestras de orina (33.76\%), 366 muestras de hisopado rectal (32.12\%), 387 muestras de hisopado faríngeo (34.03\%) y 1 muestra de hisopado uretral (0.09\%).

Las muestras colectadas por la Asociación Civil Impacta Salud y Educación (IMPACTA) en Lima y Callao, fueron transportadas a $4^{\circ} \mathrm{C}$ en tubos de colección del kit APTIMA hasta el Laboratorio de Bacteriología de la Unidad de Investigación de Enfermedades Tropicales de La Marina de Los Estados Unidos (NAMRU-6) para detectar la presencia de Neisseria gonorrhoeae como parte del estudio clínico que examina el efecto de la Terapia Expedita para Parejas (Expedited Partner Therapy, EPT) y cuyo registro de resultados clínicos de los participantes está disponible en la web con el siguiente número de accesión ClinicalTrials. gov número, NCT01720654.

\section{Procedimientos de laboratorio}

Detección de Neisseria gonorrhoeae.- Se realizó el ensayo de APTIMA Combo 2 según las instrucciones del fabricante específicamente para la detección de Neisseria gonorrhoeae. El ensayo APTIMA Combo 2 (Gen-Probe Inc., San Diego, Calif.) es una prueba de Amplificación de Ácidos Nucleicos (NAAT) que utiliza la captura de ácido ribonucleico ribosomal $(\mathrm{ARNr})$ para detección cualitativa in vitro de Neisseria gonorrhoeae y diagnóstico de enfermedades urogenitales y gonocócicas. El ensayo APTIMA Combo 2 es una NAAT de segunda generación que consta de tres pasos los cuales ocurren en el mismo tubo de reacción: aislamiento de la secuencia diana utilizando la tecnología de captura del objetivo, amplificación de la secuencia diana aislada usando tecnología de Amplificación Mediada por Transcripción (transcription-mediated amplification, TMA) y detección de señal emitida por la sonda hibridada usando el Método de Ensayo cinético dual (Dual Kinetic Assay, DKA), el cual permite la diferenciación de la señal e identificación de este patógeno.

Detección de $N$. gonorrhoeae resistente a quinolonas. - Utilizamos el Kit de extracción de ADN por columnas de centrifugación de QIAGEN (tecnología QIAamp) para aislamiento y purificación de $\mathrm{ADN}$ genómico en muestras de orina, hisopados rectales, faríngeos y uretrales positivas a Neisseria gonorrhoeae. Con el ADN se procedió a realizar PCR en tiempo real en el equipo LightCycler 2.0 (Roche Diagnostics, Tokyo, Japan). La mezcla fue $2 \mu \mathrm{L}$ de reacción comercial LightCycler FastStart DNA Master HybProbe 10X (contiene FastStart Taq ADN Polimerasa, buffer de reacción y dNTPs), $2.4 \mu \mathrm{L} \mathrm{MgCl}_{2} 10 \mathrm{mM}$, $1 \mu \mathrm{L}$ cebadores $10 \mathrm{nM}$ y $0.2 \mu \mathrm{L}$ sondas $10 \mathrm{nM}, 1 \mu \mathrm{L}$ de ADN a $100 \mathrm{ng}$ y completamos con agua hasta $20 \mu \mathrm{L}$ de volumen total. El ciclaje consistió en denaturación inicial a $95^{\circ} \mathrm{C}$ por 5 minutos, seguido de 45 ciclos de amplificación: denaturación a $95^{\circ} \mathrm{C}$ por 5 segundos, hibridación a $52^{\circ} \mathrm{C}$ por 5 segundos, y extensión a $72{ }^{\circ} \mathrm{C}$ por 10 segundos con una rampa de velocidad de $20^{\circ} \mathrm{C} / \mathrm{s}$. Después de completar el proceso de amplificación, las mezclas de reacción fueron denaturadas a $95^{\circ} \mathrm{C}$ por 0 segundos, $40{ }^{\circ} \mathrm{C}$ por 5 segundos y luego lentamente llevado a $80^{\circ} \mathrm{C}$ a una rampa de velocidad de $0.1{ }^{\circ} \mathrm{C} / \mathrm{s}$. El análisis de las curvas de Melting para determinar los especímenes mutantes y silvestres fue realizado usando el software LightCycler 2.0 software versión 3.5 (Roche Diagnostics). Los cebadores fueron diseñados para amplificar la región Determinante de Resistencia a Quinolonas (QRDR) comprendida entre los nucleótidos 174 y 306 del gen gyrA de la subunidad A de la ADN girasa (Siedner et al. 2007) y las sondas gyrA-SER91-FLU y gyrA-SER91-LC (Tabla 1) fueron usados para detectar mutaciones en el codón de la serina en posición 91 del gen gyrA mediante Temperatura de melting.

Análisis de secuencias mutantes del gen gyrA de Neisseria gonorrboeae.- Para obtener copias adicionales de la región comprendida entre los nucleótidos 174 y 306 del gen gyrA de la subunidad A de la ADN girasa hicimos PCR convencional en el termociclador VERITI 96 well Thermal Cycler (Applied Biosystems). La mezcla de reacción fue $2 \mu \mathrm{L}$ de $10 \mathrm{X}$ LightCycler FastStart DNA Master HybProbe (mezcla de reacción comercial), $2.4 \mu \mathrm{L} \mathrm{MgCl}_{2} 10 \mathrm{mM}, 1 \mu \mathrm{L}$ de cada cebador $10 \mathrm{nM}, 2 \mu \mathrm{L}$ de $\mathrm{ADN}$ a $100 \mathrm{ng}$ y completamos con agua para volumen total de $20 \mu \mathrm{L}$. Las condiciones de ciclaje fueron denaturación inicial de $95^{\circ} \mathrm{C}$ por 5 minutos, seguido de 35 ciclos de amplificación que comprende denaturación a $95^{\circ} \mathrm{C}$ por 5 segundos, hibridación a $52{ }^{\circ} \mathrm{C}$ por 5 segundos, y extensión a $72{ }^{\circ} \mathrm{C}$ por 10 segundos. Después de completar este proceso se mantuvo a $72{ }^{\circ} \mathrm{C}$ por 5 minutos para luego bajar la temperatura a $4^{\circ} \mathrm{C}$ hasta el momento de retirarlo del termociclador. Los productos de PCR fueron verificados por electroforesis en agarosa al 2\% (UltraPure ${ }^{\mathrm{TM}}$ Agarose Invitrogen y SYBR ${ }^{\circ}$ Safe DNA gel stain Invitrogen) y purificados con el kit QIAquick PCR Purification (Qiagen).

Usamos el kit de secuenciamiento BigDye Terminator v3.1 (Applied Biosystem, Foster City-United States) para que la Taq-polimerasa incorpore los dideoxinucleótidos-trifosfatos (ddNTPs). La mezcla de reacción contenía 4ul de Solución BDT V3.1, $4 \mu \mathrm{L}$ de Buffer BDT 5X, $1 \mu \mathrm{L}$ del cebador $0.1 \mu \mathrm{M}, 2 \mu \mathrm{L}$ de ADN 100 ng y completamos con agua hasta $20 \mu \mathrm{L}$. Colocamos la mezcla en el termociclador GENEAMP ${ }^{\oplus}$ SYSTEM 9700 Applied Biosystems, con el programa de amplificación siguiente: 96 ${ }^{\circ} \mathrm{C}$ durante 1 minuto $\mathrm{x} 1$ ciclo; $96^{\circ} \mathrm{C}$ durante 10 segundos, 50 ${ }^{\circ} \mathrm{C}$ durante 5 segundos y $60^{\circ} \mathrm{C}$ durante 4 minutos x 25 ciclos y

Tabla 1. Cebadores y secuencias de oligonucleótidos de sonda FRET de PCR en tiempo real

\begin{tabular}{lcc}
\hline Cebador o sonda & Secuencia oligonucleotídca & Posición \\
\hline Cebadores & & $174-194$ \\
NG-gyrA-Ser91-F & 5'-CGC-GAT-GCA-CGA-GCT-GAA-AAA-3' $^{\prime}$ & $289-306$ \\
NG-gyrA-Ser91-R & $5^{\prime}$-TTG-CGC-CAT-ACG-GAC-GAT-3' & $227-261$ \\
Sondas & & $263-279$ \\
gyrA-Ser91-Flu & 5'-GCA-TCG-TCG-GCG-ACG-TCA-TCG-GTA-AAT-ACC-ACC-C-3'-fluoresceína $^{\text {gyrA-Ser91-LC }}$ & 5'-Red 640-ACG-GCG-ATT-CCG-CAG-TT-3'-fosforilado
\end{tabular}


finalmente baja a $4^{\circ} \mathrm{C}$ por tiempo indefinido. Los amplificados fueron purificados usando columnas de Centri-Sep (Princeton Separation - Sambrook et al. 1989), la muestra fue aplicada en la columna y se centrifugó a $3000 \mathrm{rpm}$ por 2 minutos en una centrifuga al vacío Eppendorf. Las secuencias purificadas fueron resuspendidas en formamida y la secuenciación tuvo lugar en el secuenciador ABI PRISM 3130xl Genetic Analyzer (Applied Biosystems, Foster City, Estados Unidos) con las condiciones por defecto del secuenciador.

Las secuencias resultantes fueron diseñadas y almacenadas gracias al software Sequencing Analysis, mientras que el Software SeqScape fue utilizado para la detección de mutaciones; ambos forman parte del paquete de software integrado en el secuenciador ABI PRISM 3130xl Genetic Analyzer (Applied Biosystems, Foster City, Estados Unidos). Para realizar la traducción de las secuencias nucleotídicas a secuencias aminoacídicas de los datos obtenidos de la secuenciación se empleó el software Sequencher (versión 4.8). Se analizó las secuencias aminoacídicas con la ayuda del programa Bioedit (versión 7.2.6) para observar posibles mutaciones en la Región Determinante de Resistencia a Quinolonas (QRDR) del gen gyrA. El programa ClustalW (versión 2.1), que forma parte del paquete de programas de Bioedit fue utilizado para determinar las posiciones de las mutaciones identificadas mediante alineamientos múltiples de las secuencias aminoacídicas traducidas y la secuencia de referencia del gen gyrA anotado en la base de datos del GenBank con número de accesión U08817 referido por Belland et al. (1994).
En la Tabla 2 se muestra la base de datos empleada para el reconocimiento de los patrones de mutación.

\section{Resultados}

Detección de Neisseria gonorrhoeae en muestras clínicas.- De los 367 hombres que tienen sexo con hombres (HSH), 45 de ellos fueron positivos a Neisseria gonorrhoeae. De estos, 34 fueron positivos en un solo sitio anatómico (18 fueron positivos en hisopado faríngeo, 12 en hisopado rectal y 4 en orina), 9 fueron positivos en 2 sitios anatómicos (4 fueron positivos en orina e hisopado faríngeo y 5 en hisopado rectal e hisopado faríngeo) y 2 fueron positivos a $N$. gonorrhoeae en 3 sitios anatómicos (uno resultó positivo en orina, hisopado faríngeo e hisopado uretral y el otro resultó positivo en orina, hisopado faríngeo e hisopado rectal). En algunos casos se presentó que un HSH tenía más de un sitio anatómico positivo para $N$. gonorrhoeae haciendo un total de 58 muestras positivas a $N$. gonorrhoeae. Tomando en cuenta las 58 muestras positivas de los 45 HSH infectados con $N$. gonorrhoeae, se observó que el tipo de muestra con mayor número de positivos fue el hisopado faríngeo seguido del hisopado rectal.

Detección de Neisseria gonorrboeae resistente a quinolonas.- De las 58 muestras clínicas positivas encontradas en 45 HSH infectados con Neisseria gonorrhoeae, identificamos que $11 \mathrm{HSH}$ presentaron QRNG con mutaciones en la posición serina 91 de la región determinante de resistencia a quinolonas (QRDR). Las bacterias de tipo silvestre registraron temperatura

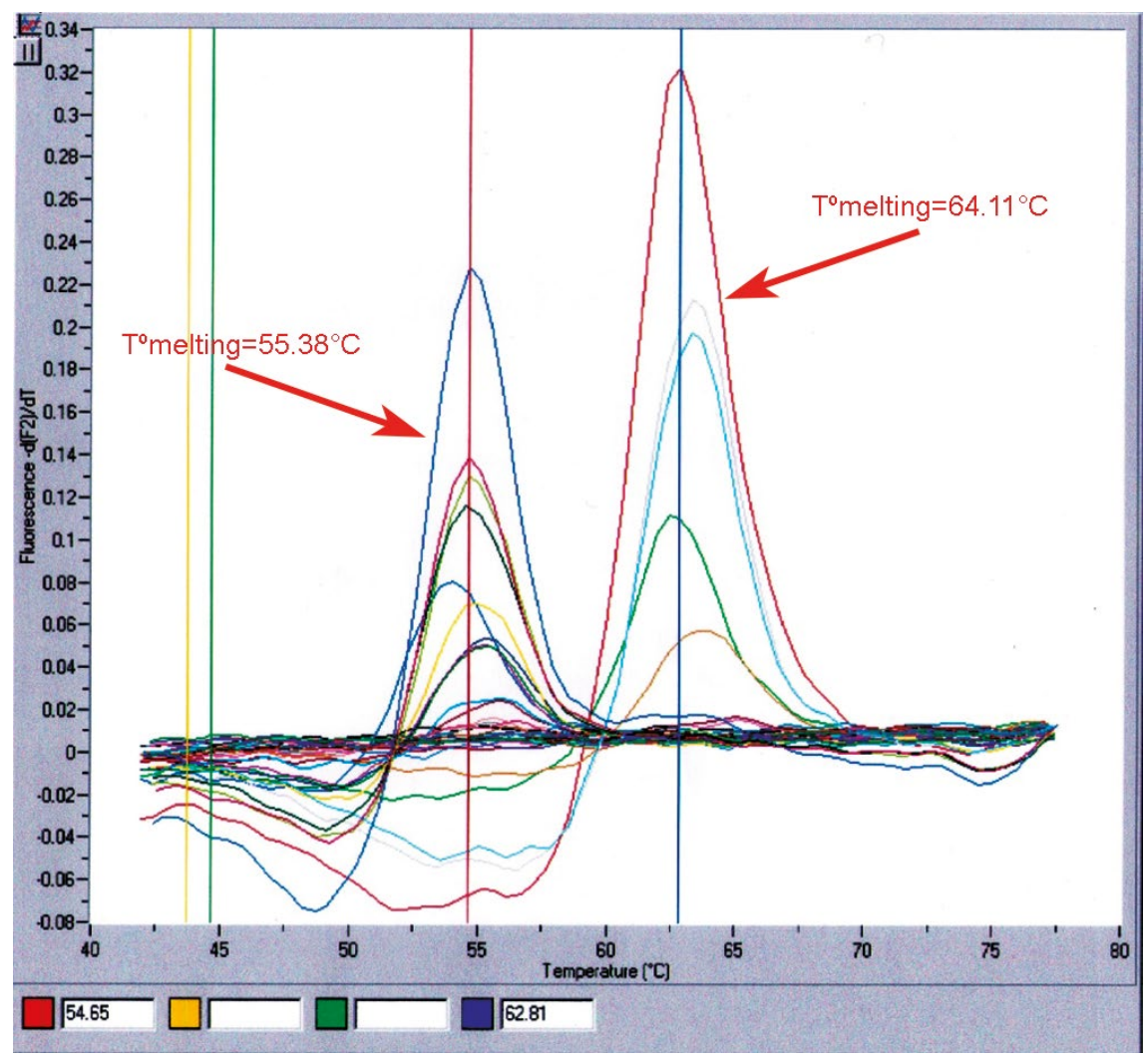

Figura 1. Curva de Melting de Neisseria gonorrhoeae fenotipo silvestre y $N$. gonorrhoeae con una substitución en la posición 91 de la Región Determinante de Resistencia a Quinolonas. Imagen tomada del equipo LightCycler 2.0 (Roche Diagnostics, Tokyo, Japan). 
Tabla 2. Base de datos de los patrones de mutación. Las secuencias QRDR muestran en negrita-subrarayado las substituciones aminoacídicas en la posición 91 y 95 de los especímenes de Neisseria gonorrhoeae con fenotipo mutante comparándolo con la primera secuencia QRDR de $N$. gonorrhoeae fenotipo silvestre.

\begin{tabular}{|c|c|c|c|c|}
\hline Año & Referencia & Localización & Secuencia QRDR & Patrones de mutación \\
\hline 1994 & Belland et al. & EE.UU. & $\begin{array}{cc}91 & 95 \\
\text { VIGKYHPHGDSAVYDTIV }\end{array}$ & Tipo Silvestre \\
\hline 1994 & Belland et al. & EE.UU. & 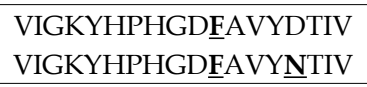 & $\begin{array}{l}\text { S91F } \\
\text { D95N }\end{array}$ \\
\hline 1995 & Deguchi et al. & Japón & 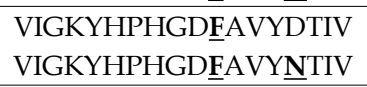 & $\begin{array}{l}\text { S91F } \\
\text { D95N }\end{array}$ \\
\hline 1996 & Deguchi et al. & Japón & 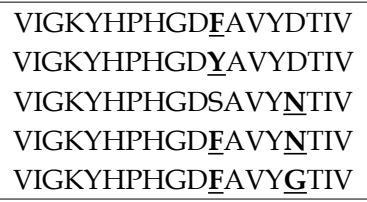 & $\begin{array}{l}\text { S91F/Y } \\
\mathrm{D} 95 \mathrm{~N} / \mathrm{G}\end{array}$ \\
\hline 1998 & Tanaka et al. & Japón & 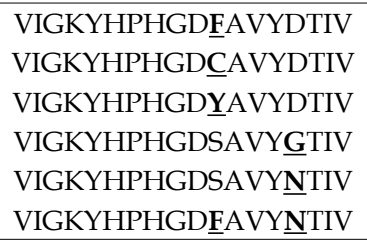 & $\begin{array}{l}\text { S91F/C/Y } \\
\text { D95N/G }\end{array}$ \\
\hline 2000 & Tanaka et al. & Japón & 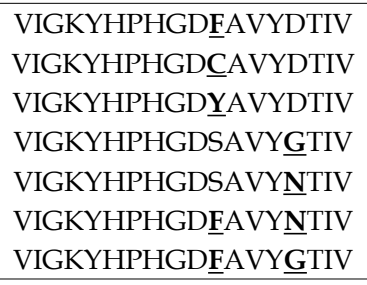 & $\begin{array}{l}\text { S91F/C/Y } \\
\text { D95N/G }\end{array}$ \\
\hline 2002 & Chaudhry et al. & India & 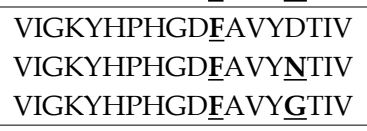 & $\begin{array}{c}\text { S91F } \\
\text { D95N/G }\end{array}$ \\
\hline 2004 & Shiguemura et al. & Japón & 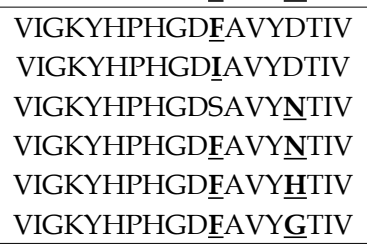 & $\begin{array}{c}\text { S91F/I } \\
\mathrm{D} 95 \mathrm{~N} / \mathrm{H} / \mathrm{G}\end{array}$ \\
\hline 2004 & Zhou et al. & China & 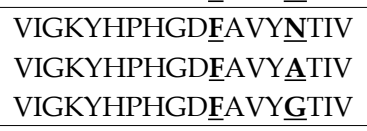 & $\begin{array}{c}\text { S91F } \\
\text { D95N/A/G }\end{array}$ \\
\hline 2005 & Lindback et al. & Suecia & 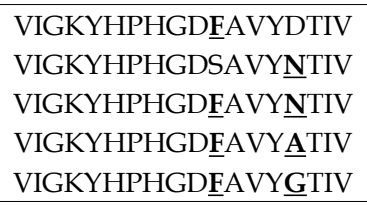 & $\begin{array}{c}\text { S91F } \\
\text { D95N/A/G }\end{array}$ \\
\hline 2009 & Zhang et al. & China & 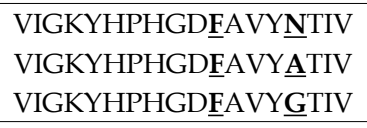 & $\begin{array}{c}\text { S91F } \\
\text { D95N/A/G }\end{array}$ \\
\hline 2009 & Zhang et al. & China & 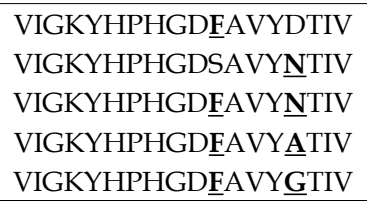 & $\begin{array}{c}\text { S91F } \\
\text { D95N/A/G }\end{array}$ \\
\hline 2011 & Uehara et al. & Brasil & 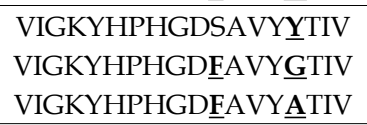 & $\begin{array}{c}\mathrm{S} 91 \mathrm{~F} \\
\mathrm{D} 95 \mathrm{Y} / \mathrm{G} / \mathrm{A}\end{array}$ \\
\hline 2012 & Kulkarni et al. & India & 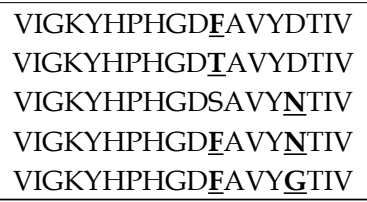 & $\begin{array}{l}\text { S91F/T } \\
\text { D95N/G }\end{array}$ \\
\hline
\end{tabular}


de melting promedio de $64.25^{\circ} \mathrm{C}+/-0.67{ }^{\circ} \mathrm{C}$; mientras que las muestras de los $11 \mathrm{HSH}$ con $N$. gonorrhoeae mutantes registraron temperatura de melting $(\mathrm{Tm})$ de $54{ }^{\circ} \mathrm{C}$ a $56.97^{\circ} \mathrm{C}$ en la QRDR (Figura 1).

En la Tabla 3 presentamos las muestras de los $11 \mathrm{HSH}$ positivos a $N$. gonorrhoeae resistente a quinolonas, el tipo de muestra y el resultado obtenido luego de realizar la PCR en tiempo real junto con su respectiva temperatura de melting (Tm). Además se muestran los resultados de $N$. gonorrhoeae con genotipo merodiploide junto con sus respectivas temperaturas de melting (Tm). Las muestras con $N$. gonorrhoeae merodiploide presentan un alelo de tipo silvestre y un alelo de tipo mutante del gen gyrA (Tobiason \& Seifert 2006), lo cual está representado en sus 2 temperaturas de melting (Tm) del PCR en tiempo real; además de las secuencias registradas como producto del secuenciamiento. El tipo de muestra más prevalente que presentó $N$. gonorrhoeae resistente a quinolona (QRNG) en los $11 \mathrm{HSH}$ fue la orina, seguida por el hisopado rectal e hisopado faríngeo. En el caso de la única muestra de hisopado uretral el resultado del PCR en tiempo real la detectó como Neisseria gonorrhoeae con genotipo silvestre.

Análisis de secuencias mutantes del gen gyrA de Neisseria gonorrboeae.- Hubieron cambios aminoacídicos en la frecuencia de patrón mutacional de las muestras de los $11 \mathrm{HSH}$ con Neisseria gonorrhoeae resistente a quinolonas (Tabla 4), siendo más frecuente la doble mutación de serina a fenilalanina en la posición 91 y de ácido aspártico a glicina en la posición 95 en un $55 \%$, mientras que la doble mutación de serina a fenilalanina en la posición 91 y de ácido aspártico a alanina en la posición 95 fue de 18\%. Adicionalmente, considerando el total de las mutaciones en la posición 91 se obtuvo que el 90.9\% (10) correspondió al cambio de serina a fenilalanina teniendo en cuenta mutaciones puntuales y dobles mutaciones y el 9.1\% (1) correspondió al cambio mutacional de serina a isoleucina. En la posición 95, considerando simples y dobles mutaciones, se obtuvo que el $54.4 \%$ (6) de las muestras cambiaron de ácido aspártico a glicina y el 18.2\% (2) substituyeron el ácido aspártico a alanina.

\section{Discusión}

En diferentes partes del mundo, la gonorrea está dentro de las infecciones de transmisión sexual con mayor frecuencia; según la OMS la incidencia anual de infecciones causadas por $N$. gonorrhoeae es de aproximadamente 128.2 millones de casos en el Pacífico Oeste seguido por 125.7 millones en las Américas y 92.6 millones en África (Soto-Cáceres 2015). En los últimos 5 años los casos de gonorrea mantienen una tendencia a incrementarse, los datos estadísticos más confiables son proporcionados por Estados Unidos de América a través del Centro de Control y Prevención de Enfermedades (CDC) que informa que cerca de 20 millones de nuevas infecciones de transmisión sexual (ITS) se presentan anualmente y de éstas la Gonorrea representa 820000 nuevos casos anuales (CDC 2013). En Estados Unidos la gonorrea es una de las enfermedades contagiosas declarada con mayor frecuencia como en el Distrito de Columbia donde la tasa en los hombres sigue siendo elevada 475 casos por cada 100000 habitantes hombres (OPS 2012).

La utilización de APTIMA y PCR en tiempo real para identificar Neisseria gonorrhoeae resistente a quinolonas en diferentes muestras se sustenta en trabajos que demuestran que las técnicas diagnósticas de amplificación de ácidos nucleicos resultan más útiles en identificación de esta bacteria comparado con el cultivo como método diagnóstico (León et al. 2016, Kirkcaldy et al. 2013, Quijano et al. 2008, CONAMUSA-MINSA 2006, Li et al. 2002).

En Perú son pocos los estudios que se han realizado sobre epidemiología de la infección por $N$. gonorrhoeae. Estos se han limitado a estimar sólo prevalencia de infección en algunos grupos de riesgo como son las trabajadoras sexuales (TS); sin embargo nuestro trabajo es el primer estudio en Perú que demuestra la presencia de Neisseria gonorrhoeae con mutaciones en la QRDR asociadas a resistencia a quinolonas en hombres que tienen sexo con hombres de Lima-Callao. El sistema de vigilancia epidemiológica tiene limitaciones que no permiten estimar la magnitud real de las ITS y no se disponen de datos oficiales; sin embargo una encuesta nacional PREVEN notificó que 1

Tabla 3. Resultados de Neisseria gonorrhoeae resistente a quinolonas (QRNG) obtenidos mediante PCR en tiempo real.

$\mathrm{TM}=$ Temperatura de melting

\begin{tabular}{ccccc}
\hline $\begin{array}{c}\mathbf{N}^{\circ} \text { de } \\
\text { muestra }\end{array}$ & $\begin{array}{c}\text { Código de } \\
\text { Laboratorio }\end{array}$ & Tipo de muestra & QRNG & TM $\left({ }^{\circ} \mathbf{C}\right)$ \\
\hline 1 & $09154-0410$ & Orina & Mutante haploide & 54 \\
2 & $09154-0429$ & Hisopado rectal & Mutante haploide & 55.15 \\
3 & $09154-0488$ & Hisopado faríngeo & Mutante haploide & 56.07 \\
4 & $09154-0526$ & Orina & Mutante haploide & 55.2 \\
5 & $09154-0542$ & Hisopado rectal & Mutante haploide & 55.2 \\
6 & $09154-0579$ & Orina & Mutante haploide & 55.2 \\
7 & $09154-0660$ & Orina & Mutante haploide & 54.53 \\
8 & $09154-0679$ & Hisopado rectal & Mutante merodiploide heterocigoto & $54.78 / 63.74$ \\
9 & $09154-0729$ & Orina & Mutante merodiploide heterocigoto & $55.77 / 65.29$ \\
10 & $09154-0744$ & Hisopado rectal & Mutante merodiploide heterocigoto & $56.32 / 65.22$ \\
11 & $09154-0761$ & Hisopado faríngeo & Mutante merodiploide heterocigoto & $56.97 / 65.34$ \\
\hline
\end{tabular}


Tabla 4. Substituciones aminoacídicas en la Región Determinante de Resistencia a Quinolonas (QRDR) del gen gyrA. Se muestran datos obtenidos del alineamiento de secuencias empleando el programa BLAST (Basic Local Alignment Search Tool). En las secuencias de aminoácidos se indica en negrita-subrayado las substituciones aminoacídicas en la posición 91 y 95 de los especímenes de Neisseria gonorrhoeae con fenotipo mutante. En los mutantes merodiploides heterocigotos se observan dos secuencias correspondientes a dos alelos del gen gyrA, una donde se observan los aminoácidos mutados en negrita-subrayado y la otra secuencia donde no hay mutación y correspondería al alelo de tipo silvestre.

\begin{tabular}{|c|c|c|c|c|c|c|c|c|c|}
\hline $\begin{array}{l}\mathrm{N}^{\circ} \text { de } \\
\text { muestra }\end{array}$ & Código & Descripción & $\begin{array}{l}\text { Max } \\
\text { Score }\end{array}$ & $\begin{array}{l}\text { Total } \\
\text { Score }\end{array}$ & $\begin{array}{l}\text { Query } \\
\text { Cover }\end{array}$ & $\begin{array}{l}\mathrm{E} \\
\text { Value }\end{array}$ & $\begin{array}{l}\text { Max } \\
\text { Ident }\end{array}$ & $\begin{array}{c}\mathbf{N}^{\circ} \text { de } \\
\text { Accesión }\end{array}$ & Traduccion \\
\hline 1 & $410 \mathrm{O}$ & $\begin{array}{l}\text { N. gonorrhoeae strain SH- } \\
\text { 8704G-Pun-2009 DNA } \\
\text { gyrase subunit A (gyrA) } \\
\text { gene, partial cds }\end{array}$ & 248 & 248 & $100 \%$ & $7 e-63$ & $100 \%$ & JX174521.1 & VIGKYHPHGD羊AVY $\underline{G T I V}$ \\
\hline 2 & $429 \mathrm{R}$ & $\begin{array}{l}\text { N. gonorrhoeae strain SH- } \\
8704 \mathrm{G}-\mathrm{Pun}-2009 \text { DNA } \\
\text { gyrase subunit A (gyrA) } \\
\text { gene, partial cds }\end{array}$ & 248 & 248 & $100 \%$ & $7 e-64$ & $100 \%$ & JX174521.2 & VIGKYHPHGDFAVY $\underline{G T I V}$ \\
\hline 3 & $488 \mathrm{~F}$ & $\begin{array}{l}\text { N. gonorrhoeae strain } \\
\text { 281G-Del-2007 DNA } \\
\text { gyrase subunit A (gyrA) } \\
\text { gene, partial cds }\end{array}$ & 132 & 132 & $96 \%$ & $5 e-28$ & $93 \%$ & JX174495.1 & VIGKYHPHGDEFAVYDTIV \\
\hline 4 & $526 \mathrm{O}$ & $\begin{array}{l}\text { N. gonorrhoeae strain SH- } \\
8704 \mathrm{G}-\mathrm{Pun}-2009 \text { DNA } \\
\text { gyrase subunit A (gyrA) } \\
\text { gene, partial cds }\end{array}$ & 248 & 248 & $100 \%$ & $7 e-63$ & $100 \%$ & JX174521.1 & VIGKYHPHGDEA VYYGTIV \\
\hline 5 & $542 \mathrm{R}$ & $\begin{array}{l}\text { N. gonorrhoeae DNA } \\
\text { gyrase subunit A (gyrA) } \\
\text { gene, gyrA-9 allele, } \\
\text { partial cds }\end{array}$ & 187 & 187 & $97 \%$ & $1 e-44$ & $100 \%$ & EU796252.1 & VIGKYHPHGD羊AVY \\
\hline 6 & $579 \mathrm{O}$ & $\begin{array}{l}\text { N. gonorrhoeae strain SH- } \\
\text { 8704G-Pun-2009 DNA } \\
\text { gyrase subunit A (gyrA) } \\
\text { gene, partial cds }\end{array}$ & 248 & 248 & $100 \%$ & $7 e-64$ & $100 \%$ & JX174521.2 & VIGKYHPHGDFAVY $\underline{\text { GTIV }}$ \\
\hline 7 & $660 \mathrm{O}$ & $\begin{array}{l}\text { N. gonorrhoeae DNA } \\
\text { gyrase subunit A (gyrA) } \\
\text { gene, gyrA-9 allele, } \\
\text { partial cds }\end{array}$ & 185 & 185 & $81 \%$ & $5 e-44$ & $100 \%$ & EU796252.1 & VIGKYHPHGD吕AVY \\
\hline \multirow{2}{*}{8} & \multirow{2}{*}{$679 \mathrm{R}$} & $\begin{array}{l}\text { N. gonorrhoeae strain SH- } \\
8704 \mathrm{G}-\mathrm{Pun}-2009 \text { DNA } \\
\text { gyrase subunit A (gyrA) } \\
\text { gene, partial cds }\end{array}$ & 248 & 248 & $82 \%$ & $9 e-63$ & $100 \%$ & JX174521.1 & VIGKYHPHGD吕AVYGGTIV \\
\hline & & $\begin{array}{l}\text { N. gonorrhoeae strain } \\
512162 \mathrm{G}-H y d-2009 \text { DNA } \\
\text { gyrase subunit A (gyrA) } \\
\text { gene, partial cds }\end{array}$ & 248 & 308 & $100 \%$ & $9 e-63$ & $100 \%$ & JX174502.1 & VIGKYHPHGDSAVYDTIV \\
\hline \multirow{2}{*}{9} & \multirow{2}{*}{7290} & $\begin{array}{l}\text { N. gonorrhoeae strain } \\
\text { 281G-Del-2007 DNA } \\
\text { gyrase subunit A (gyrA) } \\
\text { gene, partial cds }\end{array}$ & 243 & 316 & $100 \%$ & $5 e-61$ & $100 \%$ & JX174495.1 & VIGKYHPHGDEAVYDTIV \\
\hline & & $\begin{array}{l}\text { N. gonorrhoeae strain } \\
512162 \mathrm{G}-H y d-2009 \text { DNA } \\
\text { gyrase subunit A (gyrA) } \\
\text { gene, partial cds }\end{array}$ & 243 & 321 & $100 \%$ & $5 e-61$ & $100 \%$ & JX174502.1 & VIGKYHPHGDSAVYDTIV \\
\hline \multirow{2}{*}{10} & \multirow{2}{*}{$744 \mathrm{R}$} & $\begin{array}{l}\text { N. gonorrhoeae strain SH- } \\
8704 \mathrm{G}-\mathrm{Pun}-2009 \text { DNA } \\
\text { gyrase subunit A (gyrA) } \\
\text { gene, partial cds }\end{array}$ & 243 & 243 & $100 \%$ & $3 e-61$ & $99 \%$ & JX174521.1 & VIGKYHPHGD羊AVY $\underline{G T I V}$ \\
\hline & & $\begin{array}{l}\text { N. gonorrhoeae strain } \\
512162 \mathrm{G}-H y d-2009 \text { DNA } \\
\text { gyrase subunit A (gyrA) } \\
\text { gene, partial cds }\end{array}$ & 248 & 248 & $100 \%$ & $7 e-63$ & $100 \%$ & JX174502.1 & VIGKYHPHGDSAVYDTIV \\
\hline \multirow{2}{*}{11} & \multirow{2}{*}{$761 \mathrm{~F}$} & $\begin{array}{l}\text { N. gonorrhoeae strain } \\
\text { 281G-Del-2007 DNA } \\
\text { gyrase subunit A (gyrA) } \\
\text { gene, partial cds }\end{array}$ & 204 & 268 & $96 \%$ & $5 e-49$ & $100 \%$ & JX174495.1 & VIGKYHPHGDIAVYDTIV \\
\hline & & $\begin{array}{l}\text { N. gonorrhoeae strain } \\
512162 \mathrm{G}-\mathrm{Hyd}-2009 \mathrm{DNA} \\
\text { gyrase subunit A (gyrA) } \\
\text { gene, partial cds }\end{array}$ & 209 & 273 & $100 \%$ & $2 e-51$ & $95 \%$ & JX174502.1 & VIGKYHPHGDSAVYDTIV \\
\hline
\end{tabular}


a 2 de cada 100 hombres y mujeres tiene gonorrea (MINSA 2006, UPCH 2013). Nuestros resultados no coinciden con los de la encuesta PREVEN, debido a que evaluamos un grupo de riesgo en Lima-Callao registrando un valor más elevado de $45 \mathrm{HSH}$ positivos de 367; es decir 12 casos de cada 100 hombres que tienen sexo con hombres tienen gonorrea. Esto se debe a que hombres con prácticas homosexuales presentan una frecuencia elevada de infección por $N$. gonorrhoeae (Gaydos et al. 2006). Nosotros reportamos como sitio de infección más frecuente la faringe y la zona rectal, esto es válido ya que en este grupo de riesgo los sitios de infección más frecuentes son uretra, recto y faringe (Pardi et al. 2005); además la frecuencia de esta infección en hombres que tienen sexo con hombres se incrementa si estos ejercen la prostitución, encontrándose en aquellos que la ejercen frecuencias elevadas de infección uretral, rectal y faríngea (León et al. 2005, Page-Shafer et al. 2002). Según algunos autores, los hombres que tienen sexo con hombres presentan mayor incidencia de Neisseria gonorrhoeae resistente a quinolonas que los hombres heterosexuales (Kirkcaldy et al. 2013). Nosotros no presentamos valores de incidencia; pero sí un valor representativo y alarmante que 11 de $45 \mathrm{HSH}$ presentaron $N$. gonorrhoeae resistente a quinolonas en Lima-Callao, es decir 24 de cada 100 HSH.

Las quinolonas actúan inhibiendo enzimas esenciales para la replicación del ADN bacteriano, la resistencia a quinolonas ha sido asociada con alteraciones en la Región Determinante de Resistencia a Quinolonas (QRDR) de la subunidad gyrA de la ADN girasa en la posición Ser91 y Asp95 (Lindback et al. 2005). Dichas mutaciones se observan en diversos trabajos y se reconocen como marcadores de resistencia a quinolonas (Li et al. 2002, Lindbäck et al. 2005, Zhang et al. 2009). En un estudio realizado en India, las mutaciones más frecuentes fueron de serina a fenilalanina en la posición 91 (Chaudhry et al. 2002); mientras que en Japón, realizaron un estudio donde obtuvieron que el 98.8\% de las cepas QRNG presentaron una mutación en la posición 91 de serina a fenilalanina (Shigemura et al. 2004). Estos datos son semejantes a lo obtenido en nuestro trabajo donde el $90.9 \%$ de muestras clínicas de $11 \mathrm{HSH}$ presentaron la sustitución de serina a fenilalanina en posición 91 y tal sustitución está asociada a resistencia a fluoroquinolonas. Shigemura et al. también hacen referencia a una nueva mutación asociada a resistencia a fluoroquinolonas en la posición 91 con sustitución de serina a isoleucina que presenta resistencia a gatifloxacino y resistencia intermedia a ciprofloxacina; ambas mutaciones encontradas por Shigemura en la posición 91 están presentes en nuestros resultados indicando la presencia de $N$. gonorrhoeae con posible fenotipo resistente a gatifloxacino y ciprofloxacino en muestras clínicas de los $11 \mathrm{HSH}$.

Este mismo grupo de investigación también registró mutaciones asociadas a resistencia a fluoroquinolonas en la posición 95 del gen $\operatorname{gyr} A$, siendo la sustitución más frecuente de ácido aspártico a glicina en el 70.4\% de las cepas de QRNG aisladas, clasificándolas como resistentes a ciprofloxacino, levofloxacino y gatifloxacino. En nuestro análisis de secuenciamiento de muestras positivas para Neisseria gonorrhoeae también identificamos que dicha mutación en la posición 95 es la más frecuente pero con un porcentaje de $54.4 \%$ y con posible fenotipo resistente a las fluoroquinolonas mencionadas.
Existen reportes de mutaciones consideradas marcadores de resistencia a fluoroquinolonas en las posiciones serina 91 y ácido aspártico 95 de la Región Determinante de Resistencia a Quinolonas (QRDR) (Deguchi et al. 1995, Shigemura et al. 2004, Zhang et al. 2009). Deguchi et al. (1995) detectaron dobles mutaciones en la región QRDR, lo que aunado con sus datos obtenidos mediante MIC les llevó a concluir que las cepas que presentan doble mutación en la región QRDR presentan una alta resistencia a la mayoría de fluoroquinolonas como norfloxacino, ofloxacino, ciprofloxacino y fleroxacino. Nuestro análisis de secuenciamiento de la QRDR identificó dobles mutaciones en las posiciones 91 y 95 (Ser91-Phe, Ser91-Ile, Asp95-Gly, Asp95-Ala), concluyendo que las dobles mutaciones de Neisseria gonorrhoeae en nuestras muestras analizadas podrían convertir en bacterias resistentes a más fluoroquinolonas.

Las mutaciones dobles son registradas en diferentes trabajos como los de Deguchi et al. (1996) y Tanaka et al. (1998 y 2000) donde identifican dobles mutaciones como la sustitución de serina a fenilalanina en la posición 91 y la substitución de aspartato a glicina en la posición 95 de la QRDR. En nuestros resultados de secuenciamiento encontramos que dicha doble mutación fue la más prevalente hallándose en un $55 \%$ del total de muestras, esto nos podría indicar presencia de $N$. gonorrhoeae resistente a norfloxacino, levofloxacino, ciprofloxacino, esparfloxacino y otras fluoroquinolonas. Las sustituciones en la posición 91 y 95 de la Región Determinante de Resistencia a Quinolonas del gen gyrA son variadas. En aislados de Neisseria gonorrhoeae resistente a ciprofloxacino se registró sustituciones de serina a fenilalanina en la posición 91 y en la posición 95 diferentes patrones de sustitución encontradas en cepas de QRNG, de asparatato a alanina, de asparatato a glicina y de aspartato a Asparagina (Zhou et al. 2004, Lindbäck et al. 2005 y Zhang et al. 2009). Nosotros identificamos una mayor frecuencia de mutación de aspartato a glicina en la posición 95 y una mutación de menor frecuencia como la sustitución de asparatato a alanina; ambas mutaciones están muy asociadas con cepas de Neisseria gonorrhoeae resistente a ciprofloxacino.

En Brasil, la caracterización molecular de aislados de Neisseria gonorrhoeae con alta resistencia a ciprofloxacino mostraron en la QRDR del gen gyrA una mutación doble prevalente de serina a fenilalanina en la posición 91 y de aspartato a glicina en posición 95 (Uehara et al. 2011); igualmente en India se reportó un patrón similar de mutaciones (Ser91-Phe y Asp95-Gly) en aislados de Neisseria gonorrhoeae resistente a gatifloxacino, lomefloxacino, enoxacino, ciprofloxacino, norfloxacino y ofloxacino (Kulkarni et al. 2012). Nosotros identificamos un patrón similar de mutaciones en ambas posiciones de la QRDR, esto nos indica la relevancia de estas mutaciones en la resistencia de Neisseria gonorrhoeae a un mayor número de fluoroquinolonas y la utilidad de esta información para establecer recomendaciones de tratamiento efectivas dependiendo del grupo poblacional, tipo de resistencia informada y rutas de diseminación de las cepas resistentes.

Del mismo modo, mediante técnicas de caracterización molecular podemos identificar marcadores moleculares en genes gyrA y parC para estudiar, incluso dentro de la misma comunidad, la distribución geográfica de cepas gonocócicas resistentes, rutas de transmisión de las mismas y cambios fenotípicos temporales en las poblaciones bacterianas. Finalmente, es importante investigar la distribución y resistencia de Neisseria gonorrhoeae en 
poblaciones de riesgo y población abierta mediante un Sistema de Vigilancia Nacional de Gonorrea que utilice técnicas moleculares y verificación con MIC de quinolonas; ya que esta bacteria puede hacerse resistente a los antimicrobianos usados como primera línea de tratamiento debido a su alta capacidad mutagénica. Todo este análisis nos abriría la posibilidad de identificar cepas de $N$. gonorrhoeae con patrones de mutación diferentes a los observados en otras zonas geográficas, permitiendo una terapia antibiótica óptima y oportuna a los pacientes afectados para reducir los casos de QRNG.

\section{Agradecimientos}

Al Laboratorio de Bacteriología de la Unidad de Investigación de Enfermedades Tropicales de La Marina de Los Estados Unidos (NAMRU-6) por proveer las instalaciones y equipos y a la Asociación Civil IMPACTA por proveer las muestras clínicas.

\section{Literatura citada}

Arteaga B. \& Arteaga M. 2008. Infecciones Gonocócicas. Revista de la Sociedad Boliviana de Pediatría 47(2): 98-99.

Belland R. J., S. G. Morrison, C. Ison, \& W. M. Huang. 1994. Neisseria Gonorrhoeae Acquires Mutations in Analogous Regions of gyrA and parC in Fluoroquinolone-Resistant Isolates. Molecular Microbiology 14 (2): 371-380. http://dx.doi. org/10.1111/j.1365-2958.1994.tb01297.x

CDC (Centers for Disease Control and Prevention). 2006. Sexually transmitted diseases treatment guidelines, 2006. MMWR Recomm Rep;55 (RR-11):42-9. This report is available from the Internet via the CDC home page address at https://www. cdc.gov/std/treatment/2006/rr5511.pdf

CDC (Centers for Disease Control and Prevention). 2011. Sexually Transmitted Disease Surveillance. Atlanta: U.S. Department of Health and Human Services; 2012. Accessed: https:// www.cdc.gov/std/stats11/Surv2011.pdf.

CDC (Centers for Disease Control and Prevention). 2013. Incidence, Prevalence, and Cost of Sexually Transmitted Infections in the United States. CDC FACT SHEET Available in: http://www.cdc.gov/std/stats/sti-estimates-fact-sheetfeb-2013.pdf

Chaudhry U, Ray K, Bala M. et al. 2002. Mutation patterns in gyrA and parC genes of ciprofloxacin resistant isolates of Neisseria gonorrhoeae from India. Journal of Antimicrobial Chemotherapy 78:440-444. https://doi.org/10.1136/sti.78.6.440

CONAMUSA, MINSA (Ministerio de Salud), ONUSIDA, PROYECTO VIGIA. 2006. Plan Estratégico Multisectorial 2007-2011 Para la Prevención y Control de las ITS, VIH y SIDA en el PERÚ. http://www.unfpa.org.pe/ Legislacion/PDF/20070503-MINSA-Plan-MultisectorialVIH-SIDA.pdf

Deguchi T., M. Yasuda, M. Asano, K. Tada, H. Iwata, H. Komeda, T. Ezaki, I. Saito, and Y. Kawada. 1995. DNA Gyrase Mutations in Quinolone-Resistant Clinical Isolates of Neisseria Gonorrhoeae. Antimicrobial Agents and Chemotherapy 39 (2): 561-563. https://doi.org/10.1128/AAC.39.2.561.

Deguchi T., M. Yasuda, M. Nakano, S. Ozeki, T. Ezaki, I. Saito, and Y. Kawada. 1996. Quinolone-Resistant Neisseria Gonorrhoeae: Correlation of Alterations in the GyrA Subunit of DNA Gyrase and the ParC Subunit of Topoisomerase IV with Antimicrobial Susceptibility Profiles. Antimicrobial Agents and Chemotherapy 40 (4): 1020-1023.

Gaydos C.A., C.K. Kent, C.A. Rietmeijer, N.J. Willard, J.M. Marrazzo, J.B. Chapin, E.F. Dunne, et al. 2006. Prevalence of Neisseria Gonorrhoeae among Men Screened for Chlamydia Trachomatis in Four United States Cities, 1999-2003. Sexually Transmitted Diseases 33 (5): 314-319. http://dx.doi. org/10.1097/01.olq.0000194572.51186.96

Katz A.R., M.V. Lee \& G.M. Wasserman. 2012. Sexually transmitted disease (STD) update: a review of the CDC 2010 STD treatment guidelines and epidemiologic trends of common STDs in Hawai' i. Hawai' $i$ Journal of Medicine \& Public Health 71 (3):68-73. https://www.ncbi.nlm.nih.gov/pmc/ articles/PMC3313766/
Kirkcaldy R., A. Zaidi, E. Hook, et al. 2013. Neisseria gonorrhoeae antimicrobial resistance among men who have sex with men and men who have sex exclusively with women: The Gonococcal Isolate Surveillance Proyect, 2005-2010. Annals of Internal Medicine 158: 321-328. http://dx.doi. org/10.7326/0003-4819-158-5-201303050-00004

Kulkarni S., M. Bala, S. Sane, et al. 2012. Mutations in the gyrA and parC genes of quinolone-resistant Neisseria gonorrhoeae isolates in India. International Journal of Antimicrobial Agents. 40: 549 - 553. http://dx.doi.org/10.1016/j.ijantimicag.2012.08.007

Leon S., E. Segura, K. Konda, et al. 2016. High prevalence of Chlamydia trachomatis and Neisseria gonorrhoeae infections in anal and pharyngeal sites among a community-based sample of men who have sex with men and transgender women in Lima, Peru. BMJ Open 6:e008245. http://dx.doi. org/10.1136/bmjopen-2015-008245

Li Z., S. Yokoi, Y. Kawamura, et al. 2002. Rapid detection of quinolone resistance-associated gyrA mutations in Neisseria gonorrhoeae with a LightCycler. Journal of Infection and Chemotherapy 8:145-150. http://dx.doi.org/10.1007/ s101560200025

Lindbäck E., B. Gharizadeh \& F. Ataker. 2005. DNA gyrase gene in Neisseria gonorrhoeae as indicator for resistance to ciprofloxacin and species verification. International Journal of STD \& AIDS. 16: 142-147. http://dx.doi. org/10.1258/0956462053057675.

MINSA (Ministerio de Salud). 2006. Guía Nacional de Manejo de Infecciones de Transmisión Sexual. Dirección General de Salud de las Personas. Estrategia Sanitaria Nacional Prevención y Control de Infecciones de Transmisión Sexual y VIH-SIDA. Lima: MINSA. Disponible en: ftp://ftp2.minsa. gob.pe/docconsulta/documentos/dgsp/vihsida/GuiaNacionalITS_Dic2006.pdf

OPS (Organización Mundial De La Salud). 2015. Lucha contra la gonorrea resistente a los antibióticos en Tailandia. http:// www.who.int/features/2015/thailand-gonorrhoea/es/

OPS (Organización Mundial De La Salud). 2012. Salud en las Américas. Edición de 2012: Volumen de Países. Disponible en: http://www.paho.org/salud-en-las-americas-2012/index.php?option=com_docman \&task=doc_ view\&gid=217\&Itemid $=$

Page-Shafer K., A. Graves, C. Kent, et al. 2002. Increased Sensitivity of DNA Amplification Testing for the Detection of Pharyngeal Gonorrhea in Men Who Have Sex with Men. Clinical Infectious Diseases. 34:173-6. https://dx.doi. org/10.1086/338236

Pardi G., M.F. Pérez, A. Pacheco, et al. 2005. Detección de Neisseria gonorrhoeae en mucosa orofaríngea de pacientes con infección gonocóccica genital. Acta Odontologica Venezolana 43(3):228-236.

Quijano E., J. Salvatierra, V. Bedón, et al. 2008. Cultivo de gonococo positivo en Gonorrea según género y localización anatómica en el Centro de Referencia para ETS Alberto Barton. Dermatol Perú. 18(1): 19-26. http://sisbib.unmsm.edu.pe/ BVRevistas/dermatologia/v18_n1/pdf/a04v18n1.pdf

Sambrook J., E.F. Fritsch \& T. Maniatis. 1989. Molecular Cloning: A Laboratory Manual, Cold Spring Harbor Laboratory. 1626pp. http://dx.doi.org/10.1016/0092-8674(90)90210-6

Shigemura K., T. Shirakawa, H. Okada, et al. 2004. Mutations in the gyrA and parC Genes and in vitro Activities of Fluoroquinolones in 91 Clinical Isolates of Neisseria gonorrhoeae in Japan. Sexually Transmitted Diseases. 31(3):180-184. https://www.ncbi.nlm.nih.gov/pubmed/15076932

Siedner M., M. Pandori, L. Castro, et al. 2007. Real-Time PCR Assay for Detection of Quinolone-Resistant Neisseria gonorrhoeae in Urine Samples. Journal of Clinical Microbiology 45(4):1250-1254. http://dx.doi.org/10.1128/ JCM.01909-06.

Soto-Cáceres V. 2015. Infecciones de Transmisión Sexual: Epidemiología y Prevención. Rev Exp Med.; 1(2). www.rem.hrlamb. gob.pe/index.php/REM/article/download/22/20

Stefanelli P. 2011. Emerging resistance in Neisseria meningitidis and Neisseria gonorrhoeae. Expert Review of Anti-infective Therapy 9(12):1204. https://doi.org/10.1586/eri.11.146 
Tanaka M., K. Takahashi, T. Saika, et al. 1998. Development of Fluoroquinolone Resistance and Mutations involving gyrA and parC proteins among Neisseria gonorrhoeae isolates in Japan. The Journal of Urology 159:2215-2219. https://www. ncbi.nlm.nih.gov/pubmed/9598572

Tanaka M., H. Nakayama, M. Haraoka, et al. 2000. Antimicrobial Resistance of Neisseria gonorrhoeae and High Prevalence of Ciprofloxacin-Resistant Isolates in Japan, 1993 to 1998. Journal of Clinical Microbiology 38(2):521-525 https:// www.ncbi.nlm.nih.gov/pubmed/10655338

Tobiason D.M. \& H.S. Seifert. 2006. The Obligate Human Pathogen, Neisseria gonorrhoeae, Is Polyploid. PLoS BIOLOGY. 4(6):1069-1678. https://doi.org/10.1371/journal. pbio. 0040185

Uehara A., E. Amorin, M. Ferreira, et al. 2011. Molecular Characterization of Quinolone-Resistant Neisseria gonorrhoeae Isolates from Brazil. Journal of Clinical Microbiology. 49(12): 4208-4212. http://dx.doi.org/10.1128/JCM.01175-11
Unemo M. \& W. Shafer. 2011. Antibiotic resistance in Neisseria gonorrhoeae: origin, evolution, and lessons learned for the future. Annals of the New York Academy of Sciences 1230:E19-28. http://dx.doi.org/10.1111/j.1749-6632.2011.06215.x

UPCH (Universidad Peruana Cayetano Heredia). 2013. PREVEN Una estrategia efectiva para el control de las infecciones de transmisión sexual. Lima: Facultad de Salud Pública y Administración-UPCH. Disponible en: http://www. proyectopreven.org/portal/images/preven/pdf/Sistematizacion_PREVEN_DocFinal-100513.pdf

Zhang T., X. Zhou, J. Zhang, et al. 2009. Fluoroquinolone resistance among Neisseria gonorrhoeae isolates from Shanghai, China: Detection of quinolone resistance-determining region mutations. The Indian journal of medical research 129:701-706. https://www.ncbi.nlm.nih.gov/pubmed/19692753

Zhou W., W. Du, H. Cao, et al. 2004. Detection of gyrA and parC mutations associated with ciprofloxacin resistance in Neisseria gonorrhoeae by use of oligonucleotide biochip technology. Journal of clinical microbiology. 42: 5819-5824 http:// dx.doi.org/10.1128/JCM.42.12.5819-5824.2004 